\title{
Philosophiques
}

Catherine Malabou, Métamorphoses de l'intelligence. Que faire de leur cerveau bleu? Paris, Presses universitaires de France, 2017, 184 pages

\section{Ivan Bricka}

Volume 45, numéro 1, printemps 2018

URI : https://id.erudit.org/iderudit/1048631ar

DOI : https://doi.org/10.7202/1048631ar

Aller au sommaire du numéro

Éditeur(s)

Société de philosophie du Québec

ISSN

0316-2923 (imprimé)

1492-1391 (numérique)

Découvrir la revue

Citer ce compte rendu

Bricka, I. (2018). Compte rendu de [Catherine Malabou, Métamorphoses de l'intelligence. Que faire de leur cerveau bleu ? Paris, Presses universitaires de France, 2017, 184 pages]. Philosophiques, 45(1), 305-309.

https://doi.org/10.7202/1048631ar d'utilisation que vous pouvez consulter en ligne.

https://apropos.erudit.org/fr/usagers/politique-dutilisation/ 
algébriquement clos.» Ajoutons encore que la théorie des corps algébriquement clos admet l'élimination des quantificateurs (existentiels) selon un résultat de Tarski inspiré par la théorie polynomiale ou théorie des diviseurs (systèmes modulaires) de Kronecker. C'est Hermann Weyl qui sort gagnant à la fin, lui qui dans son ouvrage de 1940, Algebraic Theory of Numbers ${ }^{6}$, proclamait la supériorité de l'approche algorithmique de Kronecker sur la théorie (ensembliste) des corps (Körper) de Dedekind.

YVON GAUTHIER

Université de Montréal

\begin{abstract}
Catherine Malabou, Métamorphoses de l'intelligence. Que faire de leur cerveau bleu? Paris, Presses universitaires de France, 2017, 184 pages.
\end{abstract}

\title{
Un tournant technoscientifique, des répercussions philosophiques
}

Métamorphoses de l'intelligence. Que faire de leur cervean bleu? de Catherine Malabou est publié en août 20I7, mais reprend pour l'essentiel «trois conférences données en 2015 dans le cadre des Wellek Library Lectures à l'Université de Californie à Irvine ${ }^{1} »$. Ces communications s'articulent autour d'une réplique critique que l'auteure adresse aux propos qu'elle a elle-même tenus dans son essai Que faire de notre cerveau ?2 publié en 2004, soit avant qu'elle ne soit «réveillée d'un coup de [s]on sommeil non dogmatique ${ }^{3}$ » par des avancées significatives dans le domaine de l'intelligence artificielle.

$\mathrm{Si}$, tout comme le livre auquel il répond, Métamorphoses de l'intelligence s'inscrit dans une mouvance critique face au projet des sciences cognitives de capter scientifiquement la structure et l'activité mentales, le nouvel essai de Malabou se distingue en avouant humblement le caractère désuet de la frontière entre le biologique et l'électronique du point de vue de l'implémentation de la pensée. Aveu remarquable, faut-il le souligner, car à travers lui l'auteure concède aux partisans des approches computationnalistes en sciences cognitives que la vie dans son acception biologique n'a peut-être ni le monopole de la pensée ni celui de l'expérience, et qu'en ce sens le fonctionnalisme pourrait bien se retrouver immunisé contre les critiques lui reprochant de nier la dimension sémantique de la pensée — critiques émises

6. Hermann Weyl, Algebraic Theory of Numbers, Princeton, Princeton University Press, I998, 240 pages.

1. Catherine Malabou, Métamorphoses de l'intelligence. Que faire de leur cerveau bleu? Paris, Presses universitaires de France, 2017, p. 6.

2. Catherine Malabou, Que faire de notre cerveau? Paris, Bayard, 2004, I69 pages.

3. Catherine Malabou, Métamorphoses de l'intelligence. Que faire de leur cerveau bleu? p. IO7. 
tant par Searle ${ }^{4}$ et ses successeurs que par de nombreux continentaux, inspirés par la phénoménologie merleau-pontienne, et dont Malabou est l'héritière directe.

C'est donc le contexte technoscientifique de notre époque qui conditionne ce tournant chez Malabou. Ce contexte, par ailleurs, ne se limite pas aux bouleversements annoncés dans le domaine des modélisations de l'esprit: il se manifeste aussi dans la "gestion» de l'intelligence développée par les nouvelles technologies de télécommunication. Car s'il faut relever, dans ce livre, une force particulière, c'est bien qu'il s'intéresse à l'intelligence sous toutes ses facettes: biologique, fonctionnelle, mais aussi sociale et politique, et l'auteure se fait un devoir d'explorer, comme s'il s'agissait d'un miroir des métamorphoses de l'intelligence, les progrès techniques des deux dernières décennies tant du point de vue de ce qu'ils permettent en ce qui concerne la simulation de cognition que de celui des rapports sociaux qu'ils modifient — notamment sur le plan de la distribution et de la répartition de la connaissance.

\section{Vie de l'intelligence, intelligence de la vie}

Ces trois conférences " remaniées $^{5}$ " s'articulent autour de la notion d'intelligence sans en donner une définition claire, mais en observant plutôt comment ses acceptions ont évolué depuis les Lumières. Le parti pris méthodologique est agréablement pertinent en ce qu'il évite au lecteur d'appréhender les différentes conceptions de l'intelligence comme présentant un écart par rapport à une description objective qui en aurait été fournie d'entrée de jeu, et lui permet au contraire de saisir plus justement le point de vue de chaque paradigme sur la question de l'intelligence. La perspective historique est aussi d'autant plus adéquate que la notion d'intelligence est de nos jours considérée par plusieurs comme "floue», voire galvaudée, comme en témoignent depuis plusieurs décennies les discussions autour du concept $\mathrm{d}^{\prime}$ " intelligence artificielle ${ }^{6} »$, discussions dont nous trouvons aujourd'hui des échos dans certaines mécompréhensions des termes comme "téléphone intelligent» ou encore "ville intelligente».

L'objectif du livre est donc de présenter l'utilisation contemporaine de la notion d'intelligence par les pouvoirs scientifiques et politiques à la lumière de son histoire, d'en faire, d'une certaine manière, sa généalogie, et de comprendre le changement de paradigme qui s'opère à son sujet comme une «métamorphose» parmi d'autres.

4. Sandra Laugier et Sabine Plaud (dir.), Lectures de la philosophie analytique, Paris, Éclipses, 20 I I, p. 2 I I-229.

5. Catherine Malabou, Métamorphoses de l'intelligence. Que faire de leur cerveau bleu? p. 6.

6. Joanna Pomian, «Aux origines de l'Intelligence artificielle: H. A. Simon en père fondateur», Quaderni, vol. I, I987, p. 9-25. 
Selon Malabou, l'intelligence a subi depuis le début du $\mathrm{xx}^{\mathrm{e}}$ siècle trois métamorphoses. La première est son assimilation au déterminisme génétique et au domaine des objets mesurables. C'est par la rencontre de la biologie et de la psychologie expérimentale qu'est capturée l'intelligence: elle est objet de science, elle est déterminée, et on peut en observer les manifestations par des tests rigoureux. L'apparition des échelles de mesure, dont la plus célèbre est celle du quotient intellectuel, et la recherche du "gêne de l'intelligence" s'inscrivent alors dans la pratique des sciences normales de l'intellect. Bien entendu, cette époque de la science occidentale baigne dans un contexte politique particulièrement eugéniste, et on pourra comprendre que ces théories biologiques et psychologiques viennent renforcer une idéologie raciale et classiste qui les précédait. Ainsi, dans l'optique des tests de mesure, "les "idiots" désignent aussi bien les individus mentalement déficients que les immigrants qui arrivent à Ellis Island et se voient soumis aux tests dont ils ne comprennent pas un $\operatorname{mot}^{7}{ }$. De la même manière, la supposition d'un gène de l'intelligence consolidera le projet de politiques de "mariages judicieux » dans le but "d'améliorer la race ${ }^{8}$ ", selon les termes de la figure de proue du génétisme de l'intelligence, Francis Galton, et l'on ne connaît que trop bien les atrocités auxquelles ont abouti ces politiques.

La critique de cette métamorphose lui est contemporaine, et elle se dédouble en objections épistémologiques ${ }^{9}$ et politiques $^{10}$. Pour les détracteurs des positions déterministes de l'intelligence en génétique et en psychologie expérimentale, en effet, la mesurabilité de l'intelligence n'est pas prouvée, alors que les répercussions sociales qui sont sous-tendues par ces approches sont bel et bien observables: "On ne [mesure] jamais ainsi l'intelligence que pour la surveiller et la normaliser ${ }^{11}$. » Mais cette critique est essentiellement négative: elle ne donne pas de l'intelligence une nouvelle description qui l'affranchirait de ses limites. Qui plus est, elle "équivaut toujours à un rejet du corps ${ }^{12}$ » : en voulant abolir le déterminisme rigide dans lequel on a enfermé l'intellect, elle nie sa matérialité. C'est pour palier cette lacune que la deuxième métamorphose, celle de l'épigénétique, entre en jeu. Le paradigme épigénétique limite considérablement le rôle des gènes dans le comportement animal pour faire une plus grande place aux capacités adaptives de l'organisme dans sa totalité - et, en ce qui nous intéresse, particulièrement à celles du système nerveux. Dans cette optique, "<l'e développement psychique [...] consiste essentiellement en une marche vers

7. Catherine Malabou, Métamorphoses de l'intelligence. Que faire de leur cerveau bleu? p. 50 .

8. Ibid., p. 39.

9. Ibid., p. 6r.

10. Ibid., p. 63-72.

11. Ibid., p. 66.

12. Ibid., p. 79 . 
l'équilibre ${ }^{13}$ », vers une stabilité toujours relative à l'environnement du sujet. Le déterminisme génétique se retrouve donc significativement écarté de cette théorie, au profit du conditionnement de l'organisme, et si l'intelligence est encore "déterminée ", ce n'est ni par la naissance, qui ne lui fournit tout au plus que des conditions fondamentales de déploiement, ni de manière figée, parce que son équilibre «ne cesse jamais de se chercher puisque sa limite temporelle est indéterminée ${ }^{14} »$. Cette nouvelle conception de l'intelligence, qui se traduit matériellement par la "plasticité cérébrale», est nettement moins encadrée que la précédente, et présente en même temps l'avantage de permettre une plus grande émancipation des sujets.

De son propre aveu, jusqu'à " peu après ${ }^{15}$ » la parution de Que faire de notre cerveau?, Malabou voyait dans l'épigénétique le socle d'une pensée de l'intelligence qui permettrait de concilier le déterminisme biologique (en un sens non exclusivement génétique) et la possibilité d'une émancipation des sujets. Or, vers la fin des années 2000 , la troisième métamorphose a pointé son nez: le domaine de l'informatique a en effet été en mesure de promettre la réalisation prochaine d'une pensée libre de toute contrainte biologique, structurée uniquement par des circuits électroniques. C'est le développement, par IBM, d'une puce synaptique présentant dans une certaine mesure les capacités d'adaptation du système nerveux qui, pour l'auteure, a annoncé ce nouveau tournant. La puissance de calcul inégalée de cette nouvelle génération d'appareils, doublée de la "dé-modularisation" de leur structure, c'est-à-dire de leur conception non plus en silos communicants (mémoire, règles, entrées) mais plutôt en un tout organique dont les parties sont flexibles et reconfigurables, permettrait ainsi aux machines d'apprendre, de se reprogrammer, et d'exercer cette quête incessante d'équilibre qui caractérise l'intelligence.

Et c'est bien là tout le paradoxe auquel, ouvertement et humblement, l'essai ne parvient pas à échapper. Si l'intelligence a pu s'affranchir tour à tour de ses déterminations génétiques et de sa structure biologique, ce n'est, en fin de compte, que pour être recapturée par la machine. Et quoi de mieux que l'ordinateur pour quantifier, mesurer et déterminer? C'est paradoxalement, au bout de ce chemin d'émancipation, le projet de la génétique et de la psychologie expérimentale qui renaît. Nous n'avons néanmoins pas affaire à un déterminisme de naissance, comme le prônait Galton, ni même à une compétence générale, indépendante de son contexte d'exercice, comme l'envisageaient les partisans des échelles de mesure de l'intelligence, mais nous sommes quand même, à nouveau, devant une forme déterminée d'intelligence, qui peut être captée, contrôlée. L'intelligence artificielle, les algorithmes, le Big Data, sont autant de manifestations contemporaines de ce projet, qui unit

13. Ibid., 92

14. Ibid., p. 93 .

15. Ibid., p. I08. 
capacités cognitives et contrôle social. L'auteure n'ira pas plus loin. Elle termine l'ouvrage en assumant la nature ambivalente de l'intelligence, entre déterminisme et liberté, automatisme et autonomie, coercition et émancipation. Son dernier chapitre, "Comme un tableau de Pollock ${ }^{16}$ ", compare le mouvement de l'intelligence au geste du célèbre peintre: «ni délibéré ni hasardeux ${ }^{17}$ ", il se trouve toujours pris dans cette tension, de laquelle il est prisonnier et dont il est pourtant toujours invité à s'échapper.

\section{Un tour d'horizon simple et presque exhaustif}

À la lecture de Métamorphoses de l'intelligence, on ne peut être que convaincu de la nécessité d'une approche pluridisciplinaire pour présenter un objet aussi complexe et aussi multidimensionnel que l'intelligence. La philosophie, la psychologie, les sciences politiques, la biologie et l'informatique y sont présentées non comme des points de vue complémentaires sur la question, mais bien comme des aspects particuliers de l'intelligence, qui se font mutuellement écho et ne peuvent être pleinement compris à l'extérieur de leurs rapports d'inter-détermination.

On reprochera néanmoins à Malabou une simplicité peut-être trompeuse dans sa comparaison des systèmes nerveux et des nouveaux dispositifs informatiques. Les logiciels peuvent apprendre et se reprogrammer, mais leur support physique reste inerte et n'interagit pas avec son environnement. Et si la pensée est bien le déploiement de l'organisme, le programme n'est qu'implémenté dans les circuits électroniques. Métamorphoses de l'intelligence aurait ainsi gagné à explorer le concept de l'Umwelt, cher à la biosémiotique, qui s'est développé en parallèle des métamorphoses, à traiter de son rôle dans le raisonnement intelligent et de son vis-à-vis dans le domaine informatique - les données - , ainsi qu'à poser la question de la possibilité d'assimiler celles-ci à l'environnement d'un organisme.

Cette lacune était à souligner, mais, il faut le préciser, elle est sans doute attribuable à la simplicité et au caractère élégamment succinct de l'essai. On notera par ailleurs que, malgré son appartenance à une tradition phénoménologique, l'auteure évite d'aborder des considérations sur l'intentionnalité et l'ouverture au monde qui auraient facilement pu se montrer encombrantes et n'évoque, des écrits de Heidegger et de Derrida, que de brefs éléments incontournables au regard du sujet. Le texte réussit donc, dans une forme simple et bien synthétisée, à effectuer un tour d'horizon presque exhaustif des différentes facettes de l'intelligence du tournant du $\mathrm{xx}^{\mathrm{e}}$ siècle à aujourd'hui.

IVAN BRICKA

Université du Québec à Montréal

16. Ibid., p. I23.

17. Ibid., p. I 5 I. 